\title{
Transient Effects on Microchannel Electrokinetic Filtering with an Ion-Permselective Membrane
}

\author{
Rahul Dhopeshwarkar, ${ }^{\dagger}$ Richard M. Crooks, ${ }^{\dagger}, *$ Dzmitry Hlushkou, ${ }^{\#}$ and Ulrich Tallarek ${ }^{\S, *}$ \\ Department of Chemical Engineering, Texas A\&M University, 3122 TAMU, College Station, TX, 77843- \\ 3122, USA, and Department of Chemistry and Biochemistry, University of Texas at Austin, 1 University \\ Station, A5300, Austin, TX, 78712-0165, USA, and Institut für Verfahrenstechnik, Otto-von-Guericke- \\ Universität Magdeburg, Universitätsplatz 2, 39106 Magdeburg, Germany, and Department of Chemistry, \\ Philipps-Universität Marburg, Hans-Meerwein-Strasse, 35032 Marburg, Germany
}

* Authors to whom correspondence should be addressed.

E-mails: crooks@cm.utexas.edu; tallarek@staff.uni-marburg.de

$\dagger$ Texas A\&M University

* University of Texas at Austin

\# Otto-von-Guericke-Universität Magdeburg

$\S$ Philipps-Universität Marburg

(3 pages) 


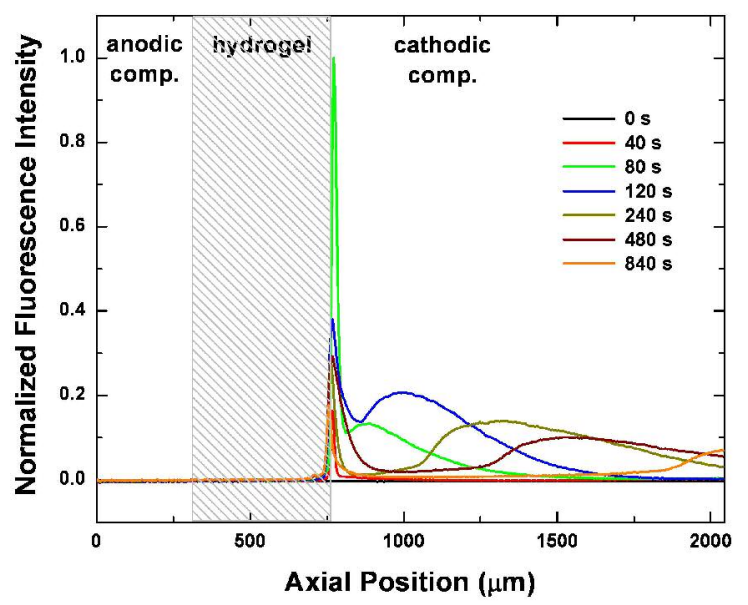

Figure S1. Fluorescence intensity profiles for 22-mer ssDNA obtained parallel to the microchannel incorporating an anionic hydrogel plug. All fluorescence intensity values were corrected by subtracting the background count before normalization. Applied potential bias, 100 V (forward).

Note: The axial position in 'pixels' has been rescaled to ' $\mu \mathrm{m}$ ' in Figure $3 \mathrm{c}$ of the main text and in Figure S1. For the images (size: 512 pixels $\times 290$ pixels) captured during the experiments described in this paper, 1 pixel equals $\sim 4 \mu \mathrm{m}$. 
Movie S1. Time-resolved fluorescence micrographs demonstrating concentration enrichment of fluorescein in a microfluidic channel incorporating an anionic hydrogel microplug. Before applying the potential bias (100 V, forward) between reservoirs ResA and ResB (Figure 2a), the buffer solution in the two reservoirs was replaced with $10.0 \mathrm{mM}$ TRIS-HCl buffer containing 5 $\mu \mathrm{M}$ fluorescein. Image frames were captured every $2 \mathrm{~s}$ for a total of 541 frames. The image size was 512 pixels $\times 290$ pixels, and the grayscale applied was 1700 to 8000 counts per pixel. The movie will play back at a rate of 10 frames/s so that the total run time is $54 \mathrm{~s}$. This movie was used to obtain the data shown in Figure 3 of the main text.

The timing for the movie is summarized in the following table.

\begin{tabular}{|c|c|c|}
\hline Show Time (s) & Real Time (s) & $\operatorname{Bias}(\mathrm{V})$ \\
\hline 0 & -60 & 0 \\
\hline 3 & 0 & 100 (forward) \\
\hline 51 & 960 & 0 \\
\hline
\end{tabular}

